\title{
Efficacy and Safety of Low Target Pressure Trabeculectomy: 2-Year Clinical Results
}

\author{
Seung Joo Ha \\ Department of Ophthalmology, Soonchunhyang University College of Medicine, Soonchunhyang University Seoul Hospital, \\ Seoul, Republic of Korea \\ Email: sjha@schmc.ac.kr
}

How to cite this paper: Ha, S.J. (2018) Efficacy and Safety of Low Target Pressure Trabeculectomy: 2-Year Clinical Results. Open Journal of Ophthalmology, 8, 42-53. https://doi.org/10.4236/ojoph.2018.81007

Received: January 11, 2018

Accepted: February 9, 2018

Published: February 12, 2018

Copyright (c) 2018 by author and Scientific Research Publishing Inc. This work is licensed under the Creative Commons Attribution International License (CC BY 4.0).

http://creativecommons.org/licenses/by/4.0/

\begin{abstract}
Purpose: To compare the success and complication rates of low target pressure trabeculectomy (LTT) and conventional trabeculectomy (CT). Methods: A retrospective study was conducted with consecutive patients undergoing trabeculectomy. Twelve eyes of 12 patients underwent LTT, and 17 eyes of 17 patients underwent CT. Surgical success was defined as meeting each target intraocular pressure (IOP) without additional medication or further glaucoma surgery. A Kaplan-Meier survival analysis was used to estimate survival rate. Incidences of surgical complications were also assessed. Results: The median postoperative IOP 2 years after surgery were $10.0 \mathrm{mmHg}$ (interquartile range [IQR] $8.5-12.0 \mathrm{mmHg}$ ) in the LTT group and $16.0 \mathrm{mmHg}$ (IQR, $14.0-18.5$ $\mathrm{mmHg})$ in the CT group $(\mathrm{P}=0.000)$. Estimated survival rates for patients who underwent the two types of trabeculectomy were significantly different with all IOP criteria of 10,12 and $14 \mathrm{mmHg}(\mathrm{P}<0.01$ for all), except IOP $\leq 18$ $\mathrm{mmHg}$. Vision-threatening complications were not found in either group during the observation period. Late-onset bleb leaks occurred in only two eyes in the LTT group but were well treated with autologous blood injection and amniotic membrane transplantation. Conclusion: LTT provided more chances to maintain low postoperative IOP and had no more vision-threatening complication than those of CT.
\end{abstract}

\section{Keywords}

Low Target Pressure Trabeculectomy, Conventional Trabeculectomy, Success Rate, Surgical Complications

\section{Introduction}

Glaucoma is an optic nerve disease and a leading cause of irreversible blindness. An increase in intraocular pressure (IOP) is the most important risk factor for 
glaucoma progression, and lowering of IOP is the most effective treatment to prevent glaucoma progression [1]. Trabeculectomy is the most popular surgical procedure for lowering IOP in patients with glaucoma refractory to medical and laser treatment, particularly primary open angle glaucoma (POAG) and normal tension glaucoma (NTG) [2].

Most trabeculectomies achieve successful outcomes and reduce IOP over the long-term, thereby minimizing progression of glaucoma [2] [3]. Maintenance of adequate aqueous outflow through the fistula is essential to prevent bleb failure. The size of the scleral flap and internal sclerostomy are the most important factors determining the amount of aqueous flow as well as the thickness of the scleral flap. Although more aqueous outflow results in a lower IOP, over filtration of the aqueous humor leads to hypotony and complications including maculopathy and choroidal detachment. It is important to make the scleral flap sufficiently large to cover the inner sclerotomy to prevent over filtration. Today, most surgeons create a 3 - 4-mm-wide half-layer scleral flap and a $1.0-1.5-\mathrm{mm}$ deep sclerostomy during the surgery [4] [5] [6] [7] [8].

However, some trabeculectomies fail to achieve long-term IOP control because of scar formation at the level of the conjunctiva tenon-episcleral interface, the scleral flap, its overlying episclera, or the sclerostomy [9] [10]. Despite increasing use of mitomycin-C or 5-fluorouracil, several studies have reported that the rate of bleb failure is $10 \%-20 \%$ [11] [12]. The most common causes of bleb failure are scar formation and closure of the fistula [13]. Bleb failure results in elevated IOP and progression of visual field (VF) loss, which are fatal to eyes with advanced glaucoma.

We have performed low target pressure trabeculectomy (LTT) in patients with advanced glaucoma to increase postoperative aqueous outflow by modifying the inner sclerostomy size and tension of the sutures to minimize bleb failure. In this study, we assessed the efficacy and safety of LTT compared with that of conventional trabeculectomy (CT) in terms of success rate and surgical complications.

\section{Materials and Methods}

Thirty-nine consecutive eyes from 34 patients who underwent trabeculectomy by an experienced glaucoma specialist (S.J.H.) between August 2010 and August 2015 were enrolled. The medical records of these patients were reviewed retrospectively. This study adhered to the tenets of the Declaration of Helsinki.

The Institutional Review Board (IRB)/Ethics Commitee approval was obtained for this retrospective study, and informed consent was obtained from all participants before surgery. We included only patients with advanced glaucoma who were diagnosed with POAG or NTG and had completed the 2-year follow up. Patients who underwent previous ophthalmic surgery except phacoemulsification were excluded.

\subsection{Visual Field Test}

Automated perimetry was conducted using the Central 24-2 Swedish Interactive 
Threshold Algorithm on a Humphrey ${ }^{\oplus}$ visual field Analyzer II (Carl Zeiss Meditec, Dublin, CA, USA) with a Goldmann size III stimulus on a 31.5-apostilb background. The VF test was performed more than two times preoperatively. Reliable VF assessment was defined as a VF test with a false-positive error < $15 \%$, a false-negative error $<15 \%$, and a fixation loss $<20 \%$. Advanced glaucoma was defined as a mean deviation (MD) of $<-12 \mathrm{~dB}$.

\subsection{Surgical Procedures}

All procedures and postoperative management were performed by the aforementioned surgeon. We created only a limbus-based conjunctival flap opening in the superonasal or superotemporal quadrant under retrobulbar anesthesia with a clear corneal traction suture. A 3-mm-wide half-layer trapezoid-shaped scleral flap was created with a crescent knife. Mitomycin C $(0.2 \mathrm{mg} / \mathrm{mL})$ was applied on and under the scleral flap and under the conjunctiva for 2 - 3 minutes.

A $1.5-\mathrm{mm}$-wide internal sclerostomy with $0.75-\mathrm{mm}$ safety margins was performed with a Kelly Descemet punch for the CT procedure used from 2010 to 2012, and a surgical iridectomy followed. The scleral flap was secured using 10-0 nylon suture, and suture tension was adjusted to allow slow oozing from the scleral flap for 4 - 5 seconds with traction.

A $2.0-\mathrm{mm}$-wide internal sclerostomy with a $0.50-\mathrm{mm}$ safety margin was performed with a Kelly Descemet punch for the LTT procedure used from 2013 to 2015, followed by surgical iridectomy (Figure 1). The scleral flap was secured using two 10-0 nylon sutures, and suture tension was adjusted to allow fast oozing from the scleral flap in 1 - 2 seconds with traction.

The conjunctival flap was sealed water tight with running locked 8-0 Vicryl sutures in all cases.

\subsection{Outcome Measures}

We assessed surgical success as achieving each IOP-related criterion as the primary outcome variable: 1 ) IOP $\leq 10 \mathrm{mmHg}, 2)$ IOP $\leq 12 \mathrm{mmHg}$, 3) IOP $\leq 14$ $\mathrm{mmHg}$, and 4) IOP $\leq 18 \mathrm{mmHg}$. Surgical success was defined as "complete success" when IOP was maintained under each criterion without an IOP-lowering procedure or additional medication, and as "qualified success" in those requiring 5-flurouracil (5-FU) needling but no additional medication.

We assessed postoperative complications including infection, symptomatic hypotony, decreased vision, maintenance of the anterior chamber, encapsulation of a bleb, and a large bleb at each follow-up visit as the secondary outcome variables. A routine postoperative examination was scheduled on day 1 , week 1 , month 1, and month 2 after surgery and then bimonthly.

\subsection{Study Groups}

The eyes were divided into two groups: the LTT group consisted of eyes that had undergone LTT, and the CT group was comprised of eyes that had undergone CT. 


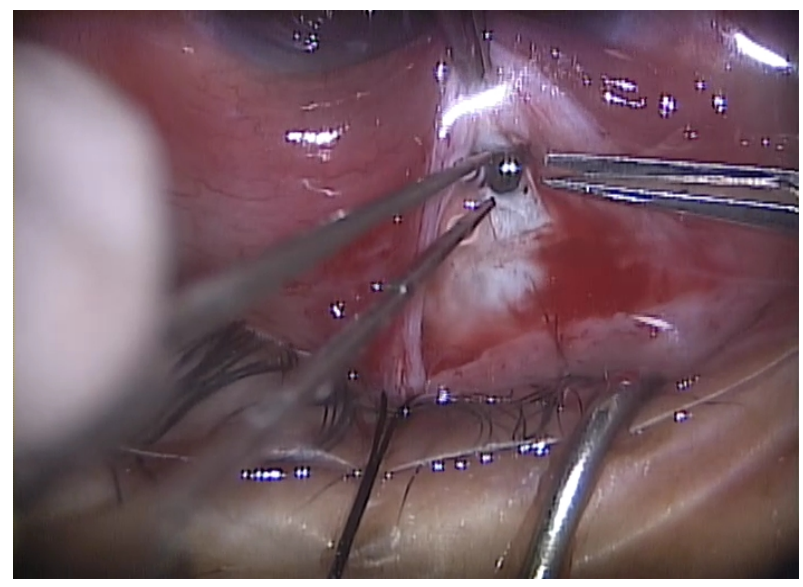

Figure 1. Intraoperative low target trabeculectomy findings. About a $2 \times 2 \mathrm{~mm}$ sized inner sclerotomy and a $0.50-\mathrm{mm}$ safety margin are shown.

\subsection{Statistical Analysis}

Wilcoxon's signed-rank test was used for comparisons between preoperative and postoperative values within the same group. The Mann-Whitney $U$-test or Fisher's exact test was used for comparisons between the LTT and CT groups. The Kaplan-Meier product-limit method was used to estimate success rate. All tests were two-tailed, and $\mathrm{P}<0.05$ was considered significant. All statistical analyses were performed with SPSS ver. 19.0 (SPSS, Inc., an IBM company, Chicago, IL, USA).

\section{Results}

\subsection{Study Population}

Twenty-seven eyes from 27 patients met the inclusion criteria and were included in the analysis. Twelve eyes from 12 patients underwent LTT, and 17 eyes from 17 patients underwent CT. No patient missed a follow-up visit during the 2-year follow-up. The preoperative mean MD values for automated perimetry were $-24.40 \pm 6.4$ and $-18.20 \pm 5.0$ in the LTT and CT groups, respectively $(\mathrm{P}=$ 0.059). No significant difference was observed between the two groups in terms of sex ratio, age, or preoperative IOP. The clinical features of the LTT and the CT groups are presented in Table 1.

\subsection{IOP and Surgical Success}

The median preoperative IOP values were $26.5 \mathrm{mmHg}$ (interquartile range[IQR] $17.0-29.8 \mathrm{mmHg}$ ) in the LTT group and $22.0 \mathrm{mmHg}$ (IQR $16.0-26.5 \mathrm{mmHg}$ ) in the CT group $(\mathrm{P}=0.444)$. The median postoperative IOP in the LTT group (7.0 mmHg, IQR $5.3-7.0 \mathrm{mmHg}$ ) was significantly lower than that in the CT group (14.0 mmHg, IQR $12.0-18.0 \mathrm{mmHg}$ ) on the first postoperative day $(\mathrm{P}<$ 0.001). Median 2-year postoperative IOP values were $10.0 \mathrm{mmHg}$ (IQR 8.5 - 12.0 $\mathrm{mmHg}$ ) and $16.0 \mathrm{mmHg}$ (IQR $14.0-18.5 \mathrm{mmHg}$ ) in the LTT and CT groups, 
Table 1. Comparisons of clinical characteristics between two groups.

\begin{tabular}{cccc}
\hline Variable & LTT group & CT group & P-value* $^{*}$ \\
\hline Number of patients & 12 & 17 & \\
Sex ratio (M/F) & $7(58.3 \%) / 5(41.7 \%)$ & $7(41.2 \%) / 10(58.8 \%)$ & 0.462 \\
Age (year) & $63.0(49.8-75.5)$ & $65.0(60.5-71.5)$ & 0.562 \\
POAG/NTG & $9(75 \%) / 3(25 \%)$ & $12(70.6 \%) / 5(29.4 \%)$ & 1.000 \\
Phakic/Pseudophakic & $10(83.3 \%) / 2(16.7 \%)$ & $14(82.4 \%) / 3(17.6 \%)$ & 1.000 \\
$\begin{array}{c}\text { Preop. IOP (mmHg) } \\
\text { Advanced glaucoma } \\
(\mathrm{MD}<-12 \mathrm{~dB})\end{array}$ & $26.50(17.0-29.8)$ & $22.0(16.0-26.5)$ & 0.444 \\
\hline
\end{tabular}

LTT = low target pressure trabeculectomy; CT = conventional trabeculectomy; $\mathrm{MD}=$ mean deviation; POAG = primary open angle glaucoma; NTG = normal tension glaucoma; $\mathrm{IOP}=$ intraocular pressure; Values are median (interquatile range) or $\mathrm{N}(\%) ;{ }^{*}$ Mann-Whitney $U$-test or Fisher's exact test.

respectively $(\mathrm{P}<0.001)$. The 2 -year postoperative IOP was significantly lower than preoperative IOP in both groups $(\mathrm{P}<0.01$, both).

The estimated survival rates of the two types of trabeculectomy were not different with the criterion of IOP $\leq 18 \mathrm{mmHg}$ in terms of complete success $(\mathrm{P}=$ $0.110)$, or qualified success $(\mathrm{P}=0.077)$. However, the LTT group revealed a higher estimated survival rate than that of the CT group with or without 5-FU needling compared with other IOP-related criteria. The differences in survival rates were maximum with criteria of IOP $\leq 12 \mathrm{mmHg}$ and IOP $\leq 14 \mathrm{mmHg}$, in terms of qualified success and complete success, respectively $(\mathrm{P}=0.000, \mathrm{P}=$ 0.002). Two patients in the LTT group and four patients in the CT group underwent 5-FU needling bleb revision during the follow-up period. The details of the success rate are presented in Figure 2 and Table 2.

\subsection{Surgical Complications}

Bleb-related infection and symptomatic hypotony did not occur during the 2 years after surgery in either group. Loss of more than three Snellen line visual acuity in the first 2 months after surgery was found in three eyes in the LTT and four eyes in the CT group $(\mathrm{P}=1.000)$. Decreased vision had improved completely in both groups until 2 months after surgery. Two eyes in the LTT group and one eye in the CT group had shallow anterior chamber depth on the first postoperative day $(\mathrm{P}=0.553)$, but all of these eyes formed a deep anterior chamber within 3 days. One eye in each group revealed bleb leakage in the first 6 weeks after surgery $(\mathrm{P}=1.000)$. Late bleb leakage was noted in two eyes in the LTT group, whereas no eye developed late bleb leakage in the CT group ( $\mathrm{P}=$ 0.163). An encapsulated bleb developed in two eyes in the LTT group and four eyes in the CT group and was treated with 5-FU needling bleb revision $(\mathrm{P}=$ $0.510)$. Overhanging blebs were noted in one eye in the LTT group and one eye in the CT group $(\mathrm{P}=0.665)$ (Table 3$)$. 

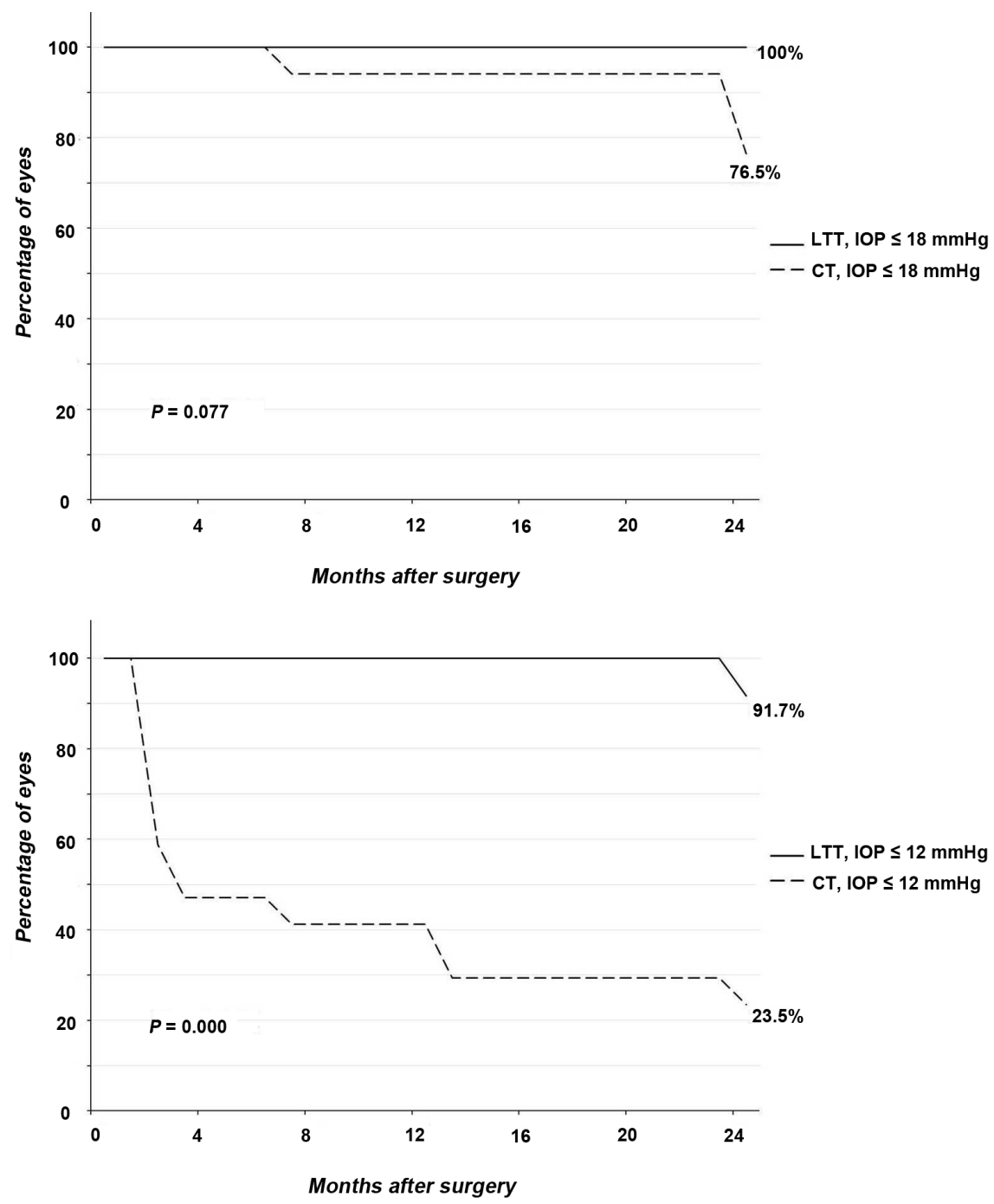

Figure 2. Kaplan-Meier estimates for the percentage of eyes with surgical success, defined as meeting the target intraocular pressure without further glaucoma surgery (qualified success). Success was achieved without additional medication, and with or without postoperative 5-flurouracil needling.

\section{Discussion}

Trabeculectomy is a widely performed glaucoma filtration surgery. Many possible complications can occur after a trabeculectomy procedure. Surgical failure is the most common complication, resulting in high postoperative IOP and subsequent progression of VF defects and blindness [3].

In the present study, the success rate in the LTT group was significantly higher than that in the CT group in terms of complete success and qualified success with each criterion. Our definition of "qualified success" was different from those of previous studies. Previous studies defined "qualified success" as surgical success with or without medication [14]. Because we performed the LTT to increase aqueous outflow through the fistula, we intended to compare the pure IOP-lowering effect of the CT and LTT. We used a different concept of 
Table 2. Surgical success in the the first 2 years after surgery.

\begin{tabular}{|c|c|c|c|c|c|c|c|}
\hline \multirow{3}{*}{ Surgical Success } & \multicolumn{4}{|c|}{ Observed \% } & \multirow{2}{*}{\multicolumn{3}{|c|}{ Survival rate ${ }^{*}$}} \\
\hline & \multicolumn{2}{|r|}{ LTT } & \multicolumn{2}{|r|}{$\mathrm{CT}$} & & & \\
\hline & Total N & Success N (\%) & Total N & Success N (\%) & LTT & $\mathrm{CT}$ & p-value \\
\hline \multicolumn{8}{|l|}{ Qualified success } \\
\hline $\mathrm{IOP} \leq 10 \mathrm{mmHg}$ & 12 & $8(66.7 \%)$ & 17 & $2(11.8 \%)$ & 0.667 & 0.118 & $0.001^{\dagger}$ \\
\hline $\mathrm{IOP} \leq 12 \mathrm{mmHg}$ & 12 & $11(91.7 \%)$ & 17 & $4(23.5 \%)$ & 0.917 & 0.235 & $0.000^{\dagger}$ \\
\hline $\mathrm{IOP} \leq 14 \mathrm{mmHg}$ & 12 & $12(100 \%)$ & 17 & $5(29.4 \%)$ & 1.000 & 0.294 & $0.001^{\dagger}$ \\
\hline $\mathrm{IOP} \leq 18 \mathrm{mmHg}$ & 12 & $12(100 \%)$ & 17 & $13(76.5 \%)$ & 1.000 & 0.765 & 0.077 \\
\hline \multicolumn{8}{|l|}{ Complete success } \\
\hline $\mathrm{IOP} \leq 10 \mathrm{mmHg}$ & 12 & $6(50.0 \%)$ & 17 & $2(11.8 \%)$ & 0.500 & 0.118 & $0.005^{\dagger}$ \\
\hline $\mathrm{IOP} \leq 12 \mathrm{mmHg}$ & 12 & $9(75.0 \%)$ & 17 & $4(23.5 \%)$ & 0.750 & 0.235 & $0.003^{\dagger}$ \\
\hline $\mathrm{IOP} \leq 14 \mathrm{mmHg}$ & 12 & $10(83.3 \%)$ & 17 & $4(23.5 \%)$ & 0.833 & 0.235 & $0.002^{\dagger}$ \\
\hline $\mathrm{IOP} \leq 18 \mathrm{mmHg}$ & 12 & $10(83.3 \%)$ & 17 & $9(52.9 \%)$ & 0.833 & 0.529 & 0.110 \\
\hline
\end{tabular}

Table 3. Surgical complications after surgery.

\begin{tabular}{|c|c|c|c|c|c|}
\hline \multirow{3}{*}{ Complications } & \multicolumn{4}{|c|}{ Observed \% } & \multirow{3}{*}{ P-value } \\
\hline & \multicolumn{2}{|r|}{ LTT } & \multicolumn{2}{|r|}{$\mathrm{CT}$} & \\
\hline & Total N & $\begin{array}{c}\text { Complication } \mathrm{N} \\
(\%)\end{array}$ & Total N & $\begin{array}{c}\text { Complication } \mathrm{N} \\
(\%)\end{array}$ & \\
\hline Infection & 12 & $0(0 \%)$ & 17 & $0(0 \%)$ & - \\
\hline Decreased vision $^{1)}$ & & & & & - \\
\hline$\leq 2$ months & 12 & $3(25.0 \%)$ & 17 & $4(23.5 \%)$ & 1.000 \\
\hline$>2$ months & 12 & $0(0 \%)$ & 17 & $0(0 \%)$ & - \\
\hline Shallow A/C & & & & & - \\
\hline$\leq 3$ days & 12 & $2(16.7 \%)$ & 17 & $1(5.9 \%)$ & 0.553 \\
\hline$>3$ days & 12 & $0(0 \%)$ & 17 & $0(0 \%)$ & - \\
\hline $\begin{array}{c}\text { Symptomatic } \\
\text { hypotony }\end{array}$ & 12 & $0(0 \%)$ & 17 & $0(0 \%)$ & - \\
\hline Bleb Leakage & & & & & - \\
\hline$\leq 6$ weeks & 12 & $1(8.3 \%)$ & 17 & $1(5.9 \%)$ & 1.000 \\
\hline$>6$ weeks & 12 & $2(16.7 \%)$ & 17 & $0(0 \%)$ & 0.163 \\
\hline Encapsulated bleb & 12 & $2(16.7 \%)$ & 17 & $4(23.5 \%)$ & 0.510 \\
\hline Overhanging bleb & 12 & $1(8.3 \%)$ & 17 & $1(5.9 \%)$ & 0.665 \\
\hline
\end{tabular}

LTT = Low target trabeculectomy; CT = Conventional trabeculectomy; A/C = anterior chamber; Decreased vision $^{1)}$ : loss of more than three Snellen lines; ${ }^{\star}$ Fishe's exact test. 
"qualified success" to exclude the IOP-lowering effect of the medication itself. Therefore, we defined "qualified success" as surgical success requiring the 5-FU needling procedure to maintain IOP below target pressure without additional medication.

The qualified success rate in the CT group was $76.5 \%$ with the IOP criterion $\leq 18 \mathrm{mmHg}$. This result is comparable with previous reports. Even if IOP remains high normal, it can lead to further optic nerve damage, VF progression, and legal blindness due to IOP fluctuations [15]. Thus, maintaining a relative low IOP is important to minimize disease progression, particularly in patients with advanced glaucoma. We performed the LTT to achieve those goals. During the operation, large inner sclerostomy and controlled scleral flap sutures allowed more aqueous flow through the fistula. LTT decreased postoperative IOP, and increased survival rate using those techniques., Postoperative IOP in the LTT group was $6.75 \pm 1.42 \mathrm{mmHg}$ on the first postoperative day and $10.25 \pm 1.872$ years after surgery. The difference in postoperative IOP between the LTT and the CT groups was significant. Postoperative IOP in the LTT group was approximately $5.8 \mathrm{mmHg}$ lower than that in the CT group.

LTT showed better efficacy than that of CT in terms of postoperative IOP. However, over filtration of the aqueous humor can lead to postoperative complications such as hypotony, a shallow anterior chamber, and late bleb leaks. De Bry et al. reported a $6.0 \%$ annual incidence of bleb leaks following trabeculectomy with mitomycin C [16]. Another study reported $10.6 \%$ incidence of symptomatic hypotony with limbus-based trabeculectomy, and that of bleb leak to be $0.4 \%$ in the first 6 weeks after surgery and $4.1 \%$ at 6 weeks after surgery. In our study, no eye developed symptomatic hypotony or a shallow anterior chamber that persisted for longer than 3 days. An early postoperative bleb leak developed in one eye in the LTT group, and one eye in the CT group. However, late-onset leak developed in only two (16.7\%) eyes in the LTT group whereas none in the CT group.

Several studies have reported treatment for late-onset bleb leaks. Hide to et al. controlled late-onset bleb leaks conservatively [17]. Autologous blood injection and amniotic membrane transplantation are frequently performed for late-onset bleb leaks [18] [19] [20]. In our cases, two eyes were treated completely with autologous blood injection and subsequent amniotic membrane transplantation (Figure 3 ). After treatment, IOPs were $<12 \mathrm{mmHg}$, and no subsequent leaks developed during the follow-up period.

Three eyes in the LTT group and four eyes in the CT group lost vision of more than three Snellen lines during the early postoperative period. However, no eye lost vision at all in the 2 months after surgery. In our study, no bleb-related infection was observed during the study period. This result was comparable with the previously reported incidence of bleb-related infection of $0.5 \%-1.0 \%$ per year [3] [21].

In our experience, various types of LTT blebs develop according to the ages and tenon tissue states of patients. Regardless of outer bleb shape, intra-bleb 


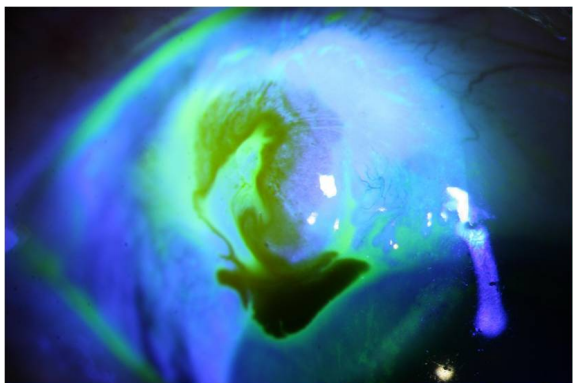

(A)

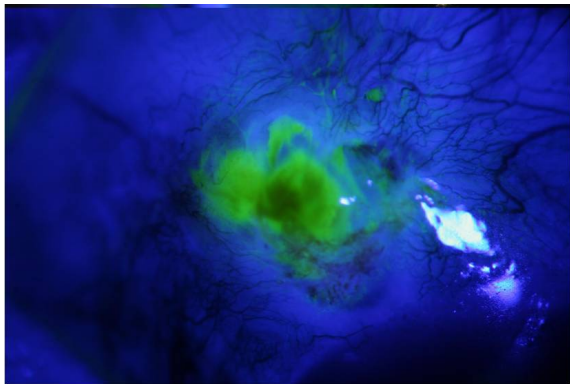

(C)

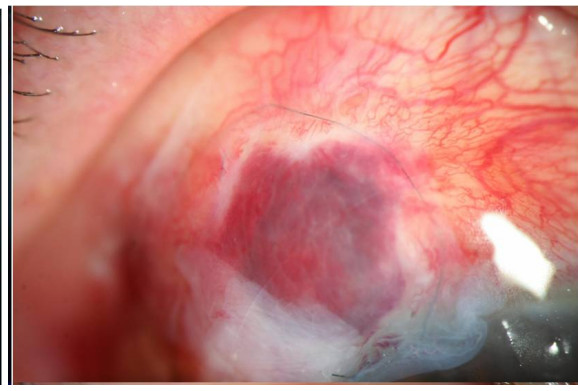

(B)

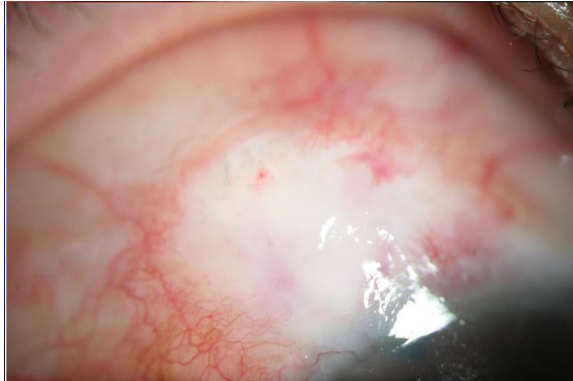

(D)

Figure 3. Autologous blood injection and amniotic membrane transplantation (AMT) for late-onset bleb leakage in a patient undergoing "low target pressure trabeculectomy". (A) Late-onset bleb leak; (B) Amniotic membrane covered the leaking bleb; (C) 2 months after AMT; (D) 4 months after AMT.

morphology of LTT blebs showed greater sub-bleb fluid collection than that of a CT bleb. The walls of a LTT bleb seemed to be thinner than those in the CT group, and revealed more multiple sponge-like microcystic changes in the conjunctiva-tenon complex (Figure 4). Because a limited number of anterior segment optical coherence tomography scans of the bleb were obtained postoperatively, we were unable to provide a statistical comparison of bleb morphology between the two groups. A morphologic analysis of the bleb may help explain the difference in postoperative IOP and the incidence of bleb-related complications between the two groups.

We have experienced several cases of blindness resulting from bleb failure in patients with advanced glaucoma before this study. This is why we performed the more aggressive surgery for patients with advanced glaucoma. This retrospective case series was too small to conclude the safety and efficacy of LTT. Despite this limitation, our study demonstrates that LTT was superior to CT in terms of maintaining low IOP all over the day and night. Furthermore, the incidence of postoperative complications was not different between the two groups, and no vision-threatening complications developed.

Another limitation of this study was the absence of a VF progression analysis. We included patients with advanced glaucoma who underwent trabeculectomy. A preoperative VF test was performed several times before surgery in all patients, but the 2-year postoperative test was absent in some patients. Therefore, we could not compare the rate of disease progression between the two groups. In 


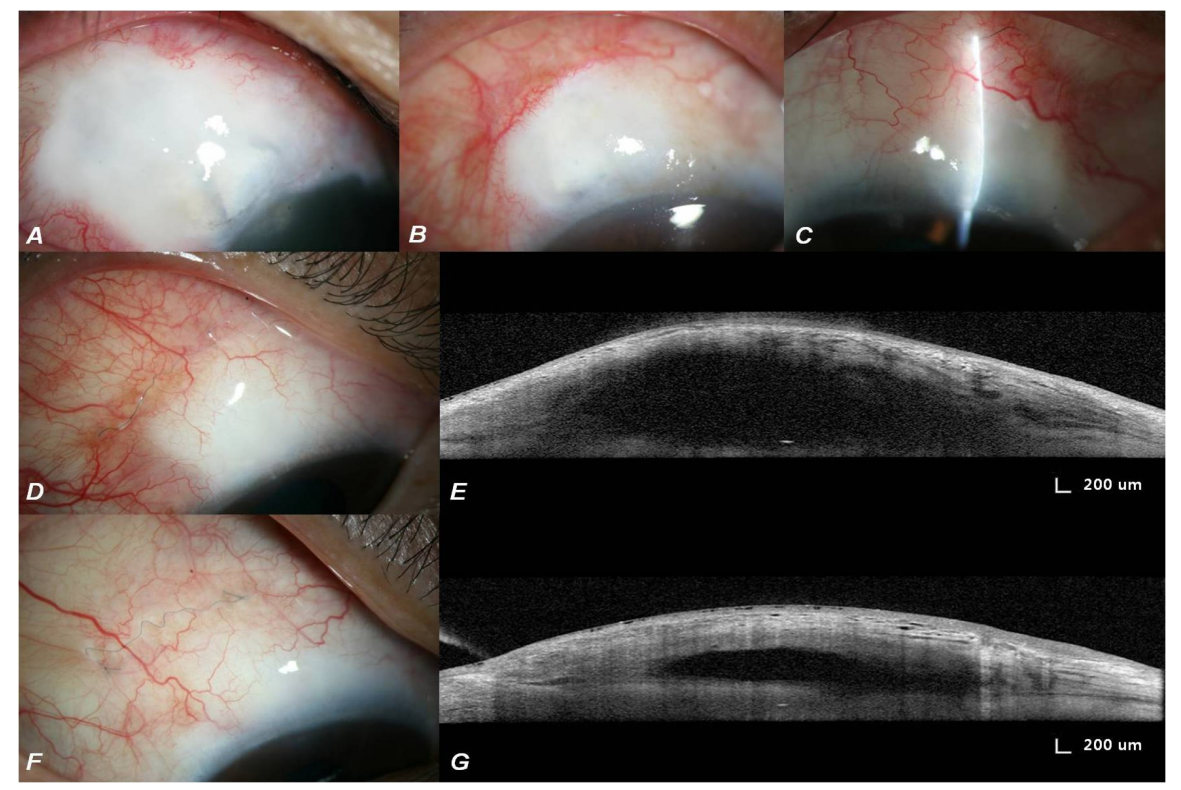

Figure 4. Various types of low target pressure trabeculectomy (LTT) blebs according to age and tenon tissue states (A)-(C). Comparison of typical diffuse, well-elevated bleb from LTT and diffuse low bleb from conventional trabeculectomy (D) (F). Representative anterior segment optical coherence tomography images of bleb from (E) "low target pressure trabeculectomy" (LTT); and (G) "conventional trabeculectomy" (CT). The bleb of the LTT has a thinner conjunctiva-tenon wall with more multiple sponge-like microcystic changes than that of the CT.

addition, the LTT group was comprised of patients with more advanced glaucoma than those in the CT group, in terms of the mean preoperative MD value. These reasons prevented us from comparing the efficacy of LTT and CT in terms of disease progression. However, we can speculate that LTT was better option able to prevent glaucoma progression than that of $\mathrm{CT}$ when we took into consideration postoperative IOP.

\section{Conclusion}

In conclusion, our results show that LTT was more efficient to lower IOP in patients with advanced glaucoma and did not compromise the safety of the surgery compared with that of CT. This aggressive surgical method is a good treatment option in patients with uncontrolled advanced glaucoma accompanied by small residual visual field.

\section{Acknowledgements}

This work was supported by the Soonchunhyang University Research Fund. The funding organization had no role in the design or conducted of this research. The author declares no competing interests.

\section{Declaration of Interest}

The author declares no conflicts of interest. 


\section{References}

[1] Gordon, M.O., Beiser, J.A., Brandt, D.J., et al. (2002) The Ocular Hypertension Treatment Study. Archives of Ophthalmology, 120, 714-720.

https://doi.org/10.1001/archopht.120.6.714

[2] Radhakrishnan, S., Quigley, H.A., Jampel, H.D., et al. (2009) Outcomes of Surgical Bleb Revision for Complications of Trabeculectomy. Ophthalmology, 116, 1713-1718. https://doi.org/10.1016/j.ophtha.2009.04.003

[3] Landers, J., Martin, K., Sarkies, N., et al. (2012) A Twenty-Year Follow-Up Study of Trabeculectomy: Risk Factors and Outcomes. Ophthalmology, 119, 694-702. https://doi.org/10.1016/j.ophtha.2011.09.043

[4] Bae, K., Suh, W. and Kee, C. (2012) Comparative Study of Encapsulated Blebs Following Ahmed Glaucoma Valve Implantation and Trabeculectomy with Mitomycin-C. Korean Journal of Ophthalmology, 26, 265-270. https://doi.org/10.3341/kjo.2012.26.4.265

[5] Stalmans, I., Gillis, A., Lafaut, A.S., et al. (2006) Safe Trabeculectomy Technique: Long Term Outcome. British Journal of Ophthalmology, 90, 44-47. https://doi.org/10.1136/bjo.2005.072884

[6] Tse, K.M., Lee, H.P., Shabana, N., et al. (2012) Do Shapes and Dimensions of Scleral Flap and Sclerostomy Influence Aqueous Outflow in Trabeculectomy? A Finite Element Simulation Approach. British Journal of Ophthalmology, 96, 432-437. https://doi.org/10.1136/bjophthalmol-2011-300228

[7] Matlach, J., Hoffmann, N., Freiberg, F.J., et al. (2012) Comparative Study of Trabeculectomy Using Single Sutures versus Releasable Sutures. Clinical Ophthalmology, 6, 1019-1027. https://doi.org/10.2147/OPTH.S32503

[8] Wells, A.P., Bunce, C. and Khaw, P.T. (2004) Flap and Suture Manipulation after Trabeculectomy with Adjustable Sutures: Titration of Flow and Intraocular Pressure in Guarded Filtration Surgery. Journal of Glaucoma, 13, 400-406. https://doi.org/10.1097/01.ijg.0000133387.82126.7c

[9] Dada, T., Kusumesh, R., Bali, S.J., et al. (2013) Trabeculectomy with Combined Use of Subconjunctival Collagen Implant and Low-Dose Mitomycin C. Journal of Glaucoma, 22, 659-662. https://doi.org/10.1097/IJG.0b013e3182594f5b

[10] Singh, K., Mehta, K., Shaikh, N.M., et al. (2000) Trabeculectomy with Intraoperative Mitomycin C versus 5-Fluorouracil. Prospective Randomized Clinical Trial. Ophthalmology, 107, 2305-2309. https://doi.org/10.1016/S0161-6420(00)00391-2

[11] Scott, I.U., Greenfield, D.S., Schiffman, J., et al. (1998) Outcomes of Primary Trabeculectomy with the Use of Adjunctive Mitomycin C. Archives of Ophthalmology, 116, 286-291. https://doi.org/10.1001/archopht.116.3.286

[12] Simsek, T., Cankaya, A.B. and Elgin, U. (2012) Comparison of Needle Revision with Subconjunctival Bevacizumab and 5-Fluorouracil Injection of Failed Trabeculectomy Bleb. Journal of Ocular Pharmacology and Therapeutics, 28, 542-546. https://doi.org/10.1089/jop.2012.0035

[13] Swan, K. (1975) Reopening of Nonfunctioning Filters-Simplified Surgical Techniques. Transactions - American Academy of Ophthalmology and Otolaryngology, 79, 342-348.

[14] Solus, J.F., Jampel, H.D., Tracey, P.A., et al. (2012) Comparison of Limbus-Based and Fornix-Based Trabeculectomy: Success, Bleb-Related Complications, and Bleb Morphology. Ophthalmology, 119, 703-711.

https://doi.org/10.1016/j.ophtha.2011.09.046 
[15] Rao, H.L., Addepalli, U.K., Jonnadula, G.B., et al. (2013) Relationship between Intraocular Pressure and Rate of Visual Field Progression in Treated Glaucoma. Journal of Glaucoma, 22, 719-724. https://doi.org/10.1097/IJG.0b013e318259b0c2

[16] DeBry, P.W., Perkins, T.W., Heatley, G., et al. (2002) Incidence of Late-Onset Bleb-Related Complications following Trabeculectomy with Mitomycin. Archives of Ophthalmology, 120, 297-300. https://doi.org/10.1001/archopht.120.3.297

[17] Sagara, H., Iida, T., Saito, K., et al. (2012) Conservative Treatment for Late-Onset Bleb Leaks after Trabeculectomy with Mitomycin C in Patients with Ocular Surface Disease. Clinical Ophthalmology, 6, 1273-1279. https://doi.org/10.2147/OPTH.S33427

[18] Burnstein, A., WuDunn, D., Ishii, Y., et al. (2001) Autologous Blood Injection for Late-Onset Filtering Bleb Leak. American Journal of Ophthalmology, 132, 36-40. https://doi.org/10.1016/S0002-9394(01)00873-X

[19] Kee, C. and Hwang, J.M. (2002) Amniotic Membrane Graft for Late-Onset Glaucoma Filtering Leaks. American Journal of Ophthalmology, 113, 834-835. https://doi.org/10.1016/S0002-9394(02)01415-0

[20] Rauscher, F.M., Barton, K., Budenz, D.L., et al. (2007) Long-Term Outcomes of Amniotic Membrane Transplantation for Repair of Leaking Glaucoma Filtering Bleb. American Journal of Ophthalmology, 143, 1052-1054. https://doi.org/10.1016/j.ajo.2007.01.016

[21] Jampel, H.D., Solus, J.F., Tracey, P.A., et al. (2012) Outcomes and Bleb-Related Complications of Trabeculectomy. Ophthalmology, 119, 712-722.

https://doi.org/10.1016/j.ophtha.2011.09.049 